\title{
Self-interference in Multi-tap Channels for Full-Duplex Wireless Systems
}

\author{
Ayman T. Abusabah ${ }^{1,2}$, Rodolfo Oliveira ${ }^{1,2}$, \\ and Luis Irio $^{2}$, \\ ${ }^{1}$ Departamento de Engenharia Electrótecnica e de Computadores, Faculdade de \\ Ciências e Tecnologia, Universidade Nova de Lisboa, Portugal \\ ${ }^{2}$ Instituto de Telecomunicações, Portugal
}

\begin{abstract}
Residual self-interference (SI) is primarily a key challenge when designing In-Band Full-duplex (IBFDX) wireless systems. Channel estimation errors are one of the major causes of residual SI. Consequently, a deeper understanding of the impact of the channel effects on the residual SI becomes indispensable. In this paper, we investigate the influence of multiple taps on the residual SI power of IBFDX systems. We first formulate the effect of having independent taps on the residual SI power mathematically. The derivations take into account the amount of interference cancellation on each tap by considering phase and amplitude estimation coefficients. We conclude that the increase in the number of taps always leads to an additive effect of the residual power. Such findings are shown mathematically and also reported in different results obtained by simulation. Finally, we compare the distribution of the residual SI power with different known distributions, concluding that Weibull and Gamma distributions are the closest ones in terms of accuracy. In-Band Full-Duplex communication Residual self-interference Independent fading taps channel.
\end{abstract}

Keywords: In-Band Full-Duplex communication · Residual self-interference Independent fading taps channel.

\section{Introduction}

In In-Band Full-Duplex (IBFDX) communications, the nodes can transmit and receive the signals simultaneously on the same frequency [1]. Compared with half-duplex communication systems, where the resources are divided between transmission and reception, the capacity of the communication link can be double in IBFDX systems [2].

The key challenge, when designing IBFDX systems, is the self-interference (SI), which is the transmitted signal being interfered with the received signal at the receiver of the same node. The SI can be mitigated by a combination of passive and active methods [3]. Passive methods use the antenna design to achieve the maximum delay spread for the SI signal. On the other hand, active methods exploit the knowledge of the SI, i.e., transmitted signal, to remove it and obtain the desired signal. Active methods are unable to eliminate the SI signal totally. This is due to the fact that the transmitted signal is being affected by channel and hardware impairments before 
reaching the receiver. Therefore, subtracting the SI signal is insufficient to suppress the residual power perfectly.

We define the residual SI as the amount of remaining signal after the cancellation of the SI signal. In order to reduce the amount of residual SI, the effects of the propagation channel have to be estimated and injected in the cancellation process before the subtraction. Therefore, it is significant to explore the effect of the propagation channel on the residual SI power. In this work, we study the behavior of the residual SI power in multiple taps channel for IBFDX systems. We characterize the power distribution for a different number of taps and cancellation errors through analytical formulation and simulation results.

\subsection{Research Question and Motivation}

The key challenge when designing the IBFDX system is to reduce the amount of residual SI. Apart from hardware impairment, the amount the residual SI power is mainly related to the accuracy of the estimated channels. Most of times, the transmitted signal is propagated over multiple paths, so, multiple copies, with different gains and phases, are combined at the receiver. The motivation of this work is to explore the effect of multiple taps channel on the residual SI power. Our focus is to understand the bottlenecks that limit the SI power from being totally eliminated when multiple taps are considered, by answering the following research questions:

- Question 1: What is the effect of having multiple taps channel on the residual SI power and how to characterize the residual SI power in such scenario?

- Question 2: What is the relationship between the number of propagation taps and the residual SI power?

- Question 3: What is the distribution of the residual SI power when considering a multiple taps channel?

- Question 4: What is the effect of gain, phase, and estimation error on the residual SI power?

\subsection{Related Work}

Despite of its importance, the characterization of the stochastic properties of the residual SI has received limited attention due to the difficulty of the mathematical modeling process [4-6]. In [4], the amount of cancellation and the strength of residual SI are computed considering a single-tap delay channel. The authors adopted a narrowband signal model to characterize the residual SI power, i.e., it is assumed that the signal time is less than the coherence time of the channel. The similarity of the residual SI distribution with known distributions was analyzed in [5] for a single-tap delay channel. In [6], the distribution of the residual SI power is also characterized for a single-tap delay channel. In particular, the proposed modeling methodology assumes that the signal takes the same value for a consecutive number of samples, since the carrier frequency (and consequently the sampling frequency) is higher than any frequency 
component of the input signal. To the best of our knowledge, our work is the first to consider a multi-tap channel scenario in IBFDX communication systems.

\subsection{Contributions}

In this work, the effect of multiple taps channel on the residual SI power in IBFDX systems is studied. First, the residual SI power considering multiple taps channel is mathematically formulated. We show that the residual SI power increases with the number of channel taps. We demonstrate that the amount of the cumulative power is a function of gain and phase estimation errors of each tap. Finally, we evaluate the similarity of the residual SI distribution with other known distributions. Such findings are supported through the mathematical analysis and simulation results.

\section{Technological Innovation for Life Improvement}

Over the last two decades wireless communications have changed our lives not only in terms of the way we live, but also on the way we interact socially and work. Two decades ago wireless communications already supported multiple mobile voice services, being a very important asset for a wide range of people, enabling permanent communication and the support of new and more active business schemes. While voice services were and still being a crucially important service, the need to support mobile information systems through wireless communications was also an important challenge. Consequently, the need of high-speed and low-latency mobile data communications services was quickly identified. These requirements are still under development and motivate the entire scientific and technological community in this field. This is the main reason why in the last two decades we witnessed the development of one of the fastest growing industries in the world.

Wireless connectivity has a huge impact in our lives by making certain tasks simpler than ever. Innovative services supported by wireless communications can be found in a plethora of fields: in public safety and security, where unexpected events (e.g. natural calamities) can be quickly communicated to people and machines, no matter where they are; in health care, where the fast dissemination of information can be helpful to improve treatments of remote rural areas; in the automation of traditional processes (e.g. autonomous driving, precision agriculture, industrial networks), where the mobility and/or lack of wired connectivity requires the existence of robust wireless communication services. Additionally to life improvement, the wireless communications drive and support important economic sectors, ranging from the commercial and industrial to agricultural, impacting the world in many important ways.

Although the importance of wireless communications is well recognized, the challenges related with their efficiency and latency are still under development. Traditionally, wireless communications were supported by half-duplex communication schemes, where uplink and downlink data flows were transmitted over different frequency bands. One of the multiple topics of research and innovation in wireless communications is the adoption of the so-called IBFDX communications, where both downlink and uplink flows are transmitted and received over the same frequency band. 
However, the shared nature of wireless channel demands for an efficient cancellation of the transmitted signal received in the antenna of the full-duplex device, which is particularly difficult to achieve because of the hardware impairments and the timevarying nature of the wireless communication channels. The work presented in this paper presents a contribution to better understand stochastic aspects of the residual SI due to cancellation errors in tapped-delay line wireless channels, which represent the inefficiency of the full-duplex cancellation schemes. We believe that the current study will effectively contribute to the design of more efficient full-duplex cancellation schemes, by handling the complexity of multi-tap channels in a more efficient way.

\section{System Model}

\subsection{In-Band Full-Duplex Canceller}

In this model, we consider a full-duplex scheme adopting an active analog canceler [4] that reduces the SI at the angular carrier frequency $\omega_{c}=2 \pi f_{c}$. The system model is depicted in Fig. 1. The channel is assumed to be a multiple taps channel with $I$ taps, thus, multiple shifted versions of the self-interfered signal $x_{s}(t)$ with different amplitudes are observed at the receiver side. Each tap, i.e., $i^{\text {th }}$ tap, is characterized by a delay $\tau_{i}$ and gain $h_{i}$. To obtain the residual SI signal $y_{\text {res }}(t)$, the estimated delay $\hat{\tau}_{i}$ and estimated gain $\hat{h}_{i}$ of each tap has to be injected to perform the cancellation [4].

\subsection{Residual Self-interference Power}

Generally, for $I$-taps channel and according to Fig. 1, the $y_{r e s}(t)$ can be written as follows

$$
y_{\text {res }}(t)=\sum_{i=1}^{I} h_{i} x_{s}\left(t-\tau_{i}\right) e^{j \omega_{c}\left(t-\tau_{i}\right)}-\sum_{i=1}^{I} \hat{h}_{i} x_{s}\left(t-\hat{\tau}_{i}\right) e^{j \omega_{c}\left(t-\hat{\tau}_{i}\right)},
$$

where channel gains are complex, i.e., $h_{i}=h_{r_{i}}+j h_{j_{i}}$, and uncorrelated, i.e., $E\left[h_{m} h_{n}\right]=E\left[h_{m}\right] E\left[h_{n}\right]$. The estimated gain is given by $\hat{h}_{i}=\epsilon_{i} h_{i}$, where $\left(1-\epsilon_{i}\right)$ is the gain estimation error of the $i^{t h}$ tap, i.e., $\epsilon=1$ when the channel is perfectly estimated and $\epsilon=0$ when estimation is totally corrupted. We also assume that $x_{s}(t)$ is a circularly-symmetric complex random signal and given by $x_{s}=x_{r}+j x_{j}$. 


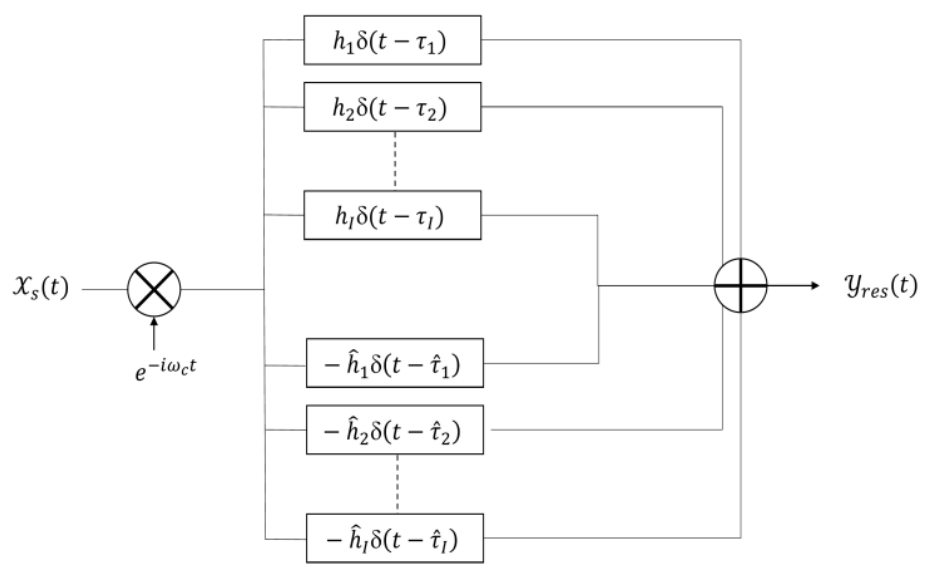

Fig. 1. Block diagram of multi-tap IBDFDX system.

Assuming a narrow band channel, i.e., $x_{s}\left(t-\tau_{i}\right)=x_{s}\left(t-\hat{\tau}_{i}\right)=x_{s}(t)$, then (1) can be represented as follows

$$
y_{\text {res }}(t)=x_{s}(t) \sum_{i=1}^{I} h_{i} c_{i},
$$

where $c_{i}=\left(e^{j \omega_{c}\left(t-\tau_{i}\right)}-\epsilon_{i} e^{j \omega_{c}\left(t-\hat{\tau}_{i}\right)}\right)$ is a constant. Since the terms $x_{s}(t), h_{i}$, and $c_{i}$ are complex, and considering that the gains of the taps are uncorrelated, then, the residual SI power can be expressed as

$$
P_{y_{r e s}}=\left(X_{r}^{2}+X_{j}^{2}\right) \sum_{i=1}^{I}\left(H_{r_{i}}^{2}+H_{j_{i}}^{2}\right) C_{i},
$$

where $C_{i}=\left(1+\epsilon_{i}^{2}-2 \epsilon_{i} \cos \left(\phi_{i}\right)\right)=\left(\left(\Re\left(c_{i}\right)\right)^{2}+\left(\mathfrak{I}\left(c_{i}\right)\right)^{2}\right)$ is also a constant which represents the power of $c_{i} . \phi_{i}=\omega_{c}\left(\tau_{i}-\hat{\tau}_{i}\right)$ is the phase estimation error of the $i^{\text {th }}$ tap.

According to (3), the residual SI power is the product of the power of the transmitted signal with the summation of scaled power gains. By definition, the power of the signal and channel gains are positive, and the constant $C_{i}$ is a non-negative constant. Thus, the residual SI power increases with the number of taps when the cancellation of each tap is not perfect. When $\phi_{i}=0$ and $\epsilon_{i}=1$, then, $C_{i}=0$ and therefore the additive power of the $i^{\text {th }}$ tap is null because it is completely cancelled. On the other hand, when $\phi_{i}=$ $\pi$ and $\epsilon_{i}=1$, then, $C_{i}=4$ and therefore the additive power is maximum. Clearly, the amount of the additive power of each single tap is a function of the gain and phase errors and the minimum value it can achieve is 0 . Consequently, each single-tap produces an additive effect on the residual SI power when it is not totally canceled. 


\section{Performance Analysis}

\subsection{Evaluation Methodology}

The evaluation is based on simulation. The system design in Fig. 1 is adopted. The IBFDX communication system is operating at a carrier frequency of $1 \mathrm{GHz}$. The residual SI power given in (3) is generated using empirical data. The values of $X_{r}$ and $X_{j}$ are sampled from Normal distributions, i.e., $X_{r} \sim \mathcal{N}\left(0, \sigma_{x}^{2}\right)$ and $X_{j} \sim \mathcal{N}\left(0, \sigma_{x}^{2}\right)$, with $\sigma_{x}^{2}=\frac{1}{2} . H_{r_{i}}$ and $H_{j_{i}}$ are sampled from independent Rayleigh distributions, i.e., $H_{r_{i}} \sim \mathcal{N}\left(0, \sigma_{h_{i}}^{2}\right)$ and $H_{j_{i}} \sim \mathcal{N}\left(0, \sigma_{h_{i}}^{2}\right)$.

To support the conclusions presented in Section 3 , we find the cumulative density function (CDF) of the residual SI power for a different number of independent taps, $I=1, \ldots, 6$. We consider different gain and phase estimation parameters to figure out the amount of interference cancellation on each tap. Finally, we seek to find the best distribution describing the residual SI power by comparing its probability density function (PDF) with well-known distributions.

\subsection{Accuracy Assessment}

First, we evaluate the fact that the increase in the number of taps always leads to an additive effect of the residual power. To achieve that, we simulate (3) for $I=2,3,4,5$ and 6 independent Rayleigh fading taps, with $\sigma_{h_{i}}^{2}=\frac{1}{2}$ for all $i$. Fig. 2 plots the CDF of each $P_{y_{\text {res }}}$ for different number of taps, $I$, and adopting different channel's gain estimation values $(\epsilon=1$ and $\epsilon=0.9)$. The phase estimation errors adopted in this simulations are given by $\phi_{1}=\frac{\pi}{6}, \phi_{2}=\frac{\pi}{3}, \phi_{3}=\frac{2 \pi}{3}, \phi_{4}=\frac{7 \pi}{6}, \phi_{5}=\frac{5 \pi}{3}$ and $\phi_{6}=\frac{8 \pi}{9}$. As shown in Fig. 2, the increase in the number of taps produces an additive effect on the residual power. The amount of the additive power varies according to the gain and phase estimation error.

According to (3), the amount of the accumulative power depends on $C_{i}$, which is a function of gain and phase estimation errors. To support this conclusion, we simulate the residual SI power trying to achieve the minimum additive power, i.e., $C_{i}=0$ when $\epsilon_{i}=1$ and $\phi_{i}=0$. Fig. 3 depicts the CDFs of the residual SI power for $I=2,3,4,5$ and 6. Perfect gain estimation error, i.e., $\epsilon_{i}=1$, is utilized for all taps and the following phase estimation errors are adopted, $\phi_{1}=\frac{\pi}{6}, \phi_{2}=0, \phi_{3}=\frac{2 \pi}{3}, \phi_{4}=0, \phi_{5}=\frac{5 \pi}{3}$ and $\phi_{6}=0$. As seen in Fig. 3, the amount of the residual SI power for 2 taps is identical to the residual SI power of 1 tap. Also, the amount of the residual SI power for 4 and 6 taps is identical to the residual SI power of 3 and 5 taps, respectively. This is due to the fact that for $I=2,4$, and 6 , the additive power is almost null, mainly because of the predefined values of gain and phase estimation errors. 
Finally, we evaluate the similarity of the residual SI power with other known distributions. The comparison was done adopting the empirical simulated data

obtained

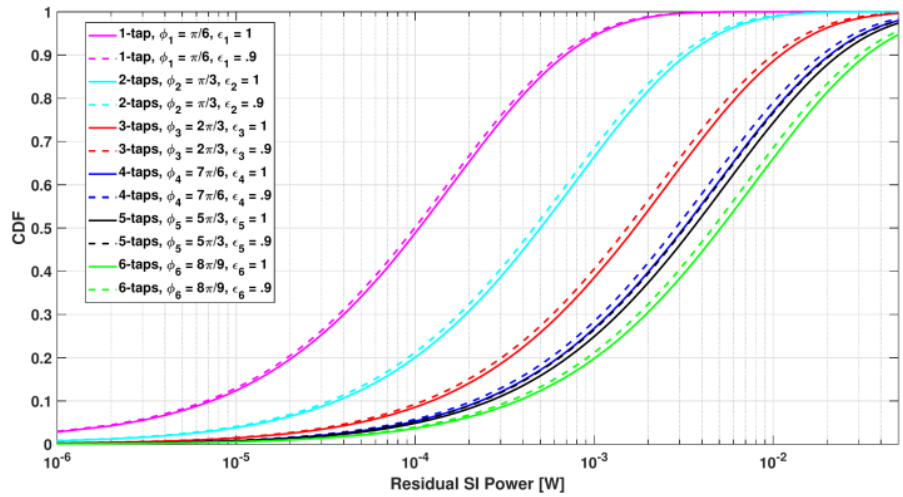

Fig. 2. CDF of residual SI power for $I=2,3,4,5$ and 6 independent Rayleigh fading channels.

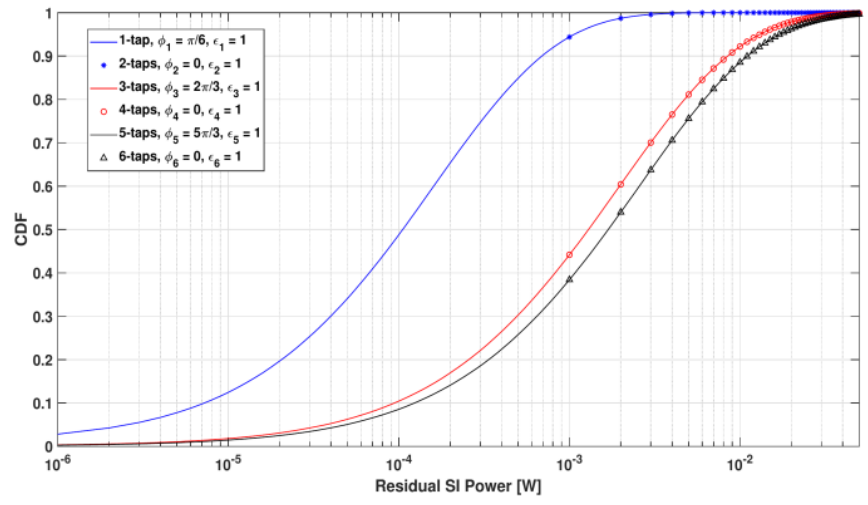

Fig. 3. CDF of residual SI power for $\mathrm{I}=2,3,4,5$ and 6 independent Rayleigh fading channels.

from the distributions plotted in Fig. 2 for 2 and 6 taps $\left(\epsilon_{i}=1\right.$ for all $\left.i\right)$. The empirical data was used in a maximum log likelihood estimation process to determine the parameters of the known distributions. The following known distributions were compared: Gamma, Exponential, Nakagami, Rayleigh, Weibull, and Lognormal. Fig. 4 plots the PDF of the two distributions that exhibited the best accuracy (Weibull and Gamma) against the PDF of the empirical data. As can be seen, Gamma and Weibull distributions exhibit almost the same level of accuracy. However, they fail to represent the probability density values at particular regions of the domain, which is even more significant as the number of taps increases. The similarity comparison clearly shows that the chosen distributions are not capable of fitting the empirical data in an accurate way over all the domain. 


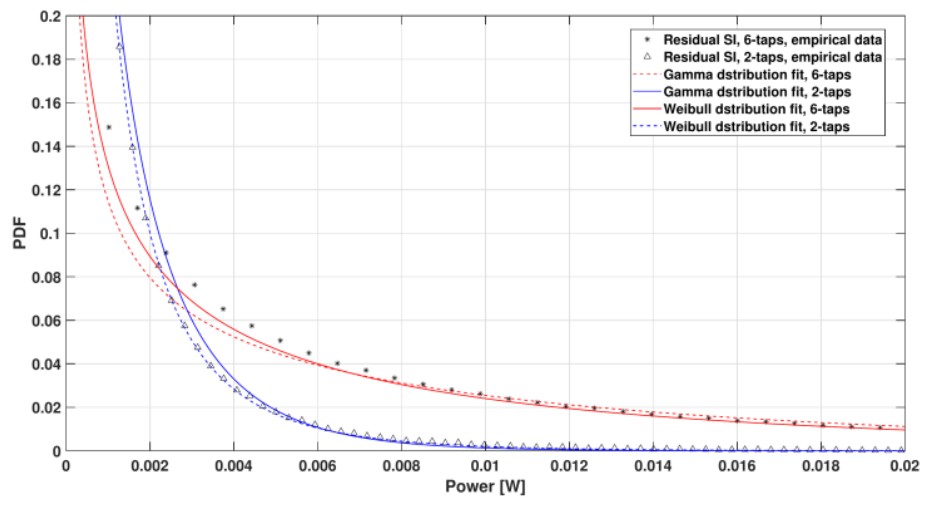

Fig. 4. Comparison of the PDF of residual SI power with other known distributions.

\section{Conclusions}

\subsection{Applicability of The Proposed Work}

The conclusions of the distribution of the residual SI power presented in this paper may be used to provide technical criteria for alleviating the SI residual interference in practical IBFDX communication systems. One of the practical applications is the compensation of the cancelation errors, i.e., the gain cancelation error $(1-\epsilon)$ and the phase cancellation error $(\phi)$. Moreover, the obtained results can also be helpful for the academic community in general, to determine different aspects related to the performance analysis of IBFDX communications. For example, by using the residual SI power to derive the outage probability of a specific FDX system, the capacity of IBFDX communication systems can be achieved.

\subsection{Final Comments}

In this paper, we have investigated the influence of multiple taps on the residual SI power of IBFDX systems. We have shown that the increase of the number of taps always leads to an additive effect of the residual power. Finally, we compared the distribution of the residual SI power with different known distributions, concluding that Gamma and Weibull only exhibit high accuracy at specific regions of the domain and a more accurate solution is desirable. Finally, we highlight that our work can be an initial step for exploring the theoretical distribution of residual SI power in multiple tap channel. The current work compared the residual SI power distribution with know distributions. Deriving the exact distribution may be considered as a future work. 
Acknowledgments. This work is supported by European Union's Horizon 2020 research and innovation programme under the Marie Sklodowska-Curie project number 813391 (TeamUp5G).

\section{References}

1. M. Heino, D. Korpi, T. Huusari, E. Antonio-Rodriguez, S. Venkatasubramanian, T. Riihonen, L. Anttila, C. Icheln, K. Haneda, R. Wichman, and M. Valkama. Recent advances in antenna design and interference cancellation algorithms for in-band full duplex relays. IEEE Commun. Mag., 53(5):91-101, May 2015

2. X. Xie and X. Zhang. Does full-duplex double the capacity of wireless networks? In Proc. IEEE INFOCOM, pages 253-261, Toronto, ON, Canada, Apr. 2014

3. A. Masmoudi and T. Le-Ngoc. A Maximum-Likelihood Channel Estimator for SelfInterference Cancelation in Full-Duplex Systems. IEEE Trans. Veh. Technol., 65(7):51225132, Jul. 2016

4. A. Sahai, G. Patel, C. Dick, and A. Sabharwal. On the impact of phase noise on active cancelation in wireless full-duplex. IEEE Trans. on Veh. Technol., 62(9):4494-4510, Nov 2013

5. L. Irio and R. Oliveira. On the impact of fading on residual self-interference power of in-band full-duplex wireless systems. In 2018 14th International Wireless Communications Mobile Computing Conference (IWCMC), pages 142-146, June 2018

6. L. Irio and R. Oliveira. Distribution of the residual self-interference power in in-band fullduplex wireless systems. IEEE Access, 7:57516-57526, 2019 